\title{
Collisional destruction experiment of chondrules and formation of fragments in the solar nebula
}

\author{
Toshihiro Ueda ${ }^{1}$, Yuuki Murakami ${ }^{1}$, Naoki Ishitsu ${ }^{1}$, Hiroshi Kawabe ${ }^{2}$, Ryuuichi Inoue ${ }^{1}$, \\ Tomoki Nakamura ${ }^{1}$, Minoru Sekiya $^{1}$, and Nobuo Takaoka ${ }^{1}$ \\ ${ }^{1}$ Department of Earth and Planetary Sciences, Graduate School of Sciences, 33 Kyushu University, Hakozaki, Fukuoka 812-8581, Japan \\ ${ }^{2}$ Department of Aeronautics and Space Engineering, Graduate School of Engineering, Tohoku University, \\ Aoba-yama 01, Sendai 980-8579, Japan
}

(Received February 21, 2001; Revised June 25, 2001; Accepted July 5, 2001)

\begin{abstract}
Collisional destruction experiments with chondrules from the Allende CV3 chondrite were performed over a range of velocities $(10 \mathrm{~m} / \mathrm{s}$ to $76 \mathrm{~m} / \mathrm{s})$. Electron microscopy shows that two types of chondrules were affected by low-velocity impacts: (1) reactivated pre-existing cracks filled with iron-oxides and (2) poorly crystallized finegrained silicates in glass. The relatively-well crystallized chondrules were destroyed at higher impact velocities. Based on the range of velocities causing chondrule destruction, we theoretically examined the condition of the solar nebula in the chondrule destruction periods and suggest that collisional destruction of chondrules can occur during abrupt and/or localized strong turbulence, in a nebular shock, by a collision between a chondrule and an object larger than $1 \mathrm{~m}$ in the laminar solar nebula.
\end{abstract}

\section{Introduction}

Chondrules are silicate spherules whose average diameter is approximately $1 \mathrm{~mm}$. They are supposed to form from molten dust particles by rapid cooling because they commonly contain glass with variable composition (e.g., Hewins, 1991). Chondrules are a major constituent in all chondritic meteorites except CI chondrites, and thus they were likely abundant in the solar nebula (Wasson, 1974). As a result, chondrules recorded some of the dynamic processes, which took place in the early solar nebula. Chondrites contain both isolated, individual chondrules and compound chondrules (Gooding and Keil, 1981). The texture of some compound chondrules indicate that they formed during collisions in the solar nebula between solid and hightemperature plastic chondrules. The presence of compound chondrules implies that the density of chondrules in the nebula was relatively high and that chondrules had relative velocities of at most $10 \mathrm{~m} / \mathrm{s}$ as they formed (Gooding and Keil, 1981; Sekiya and Nakamura, 1996).

Fragments of chondrules are common in chondrites (Steele, 1988). The destruction of chondrules could occur in the solar nebula, while chondrules were floating individually and subsequently within the meteorite parent body. Many chondrites are brecciated as a results of impacts between the meteorite parent bodies (Bunch and Rajan, 1988). During impacts most of the chondrules are broken into pieces. Therefore, it is evident that high-velocity impacts on meteorite parent bodies result in the destruction of chondrules. On the other hand, unbrecciated chondrites also contain broken chondrules, some of which are coated by fine-grained

Copy right (C) The Society of Geomagnetism and Earth, Planetary and Space Sciences (SGEPSS); The Seismological Society of Japan; The Volcanological Society of Japan; The Geodetic Society of Japan; The Japanese Society for Planetary Sciences. rims (Fig. 1). Such meteorites exhibit few impact effects (Scott et al., 1992) and the fine-grained rims may have formed, while the chondrule floated in the thick dust clouds of the nebula (e.g., Metzler et al., 1992). Therefore, it is suggested that broken chondrules with rim have experienced dynamic events in the nebula prior to rim formation. In the present study, we examine the possibility of chondrule destruction due to impacts in the solar nebula. Collisional destruction experiments were performed on the chondrules removed from the Allende CV 3 carbonaceous chondrite, in order to know the range of impact velocities over which chondrules are broken. Then, we can theoretically explore the conditions of the solar nebula where chondrules can collide each other with relative velocities high enough to destroy them.

\section{Experiment \\ 2.1 Samples}

Using a stereoscope and edged tool, 131 chondrule samples were selected from chips of the Allende CV3 chondrite. We used chondrules from Allende, because they do not have extensive secondary alteration, such as aqueous alteration and shock and thermal metamorphism (Weisberg and Prinz, 1998; Clayton and Mayeda, 1999). The Diameter and mass of each chondrule used in the experiments is summarized in Table 1.

\subsection{Method}

2.2.1 Velocity calibration A rubber gun was used for low velocity impact experiments from 10 to $30 \mathrm{~m} / \mathrm{s}$ and an air gun was used for high velocity from 45 to $150 \mathrm{~m} / \mathrm{s}$. The rubber gun consists of a launcher (Fig. 2), a sample recovery apparatus with a stainless steel target, and a camera. First, glass spherules with diameters of $5 \mathrm{~mm}$ and densities of 
Table 1. Longest dimension of major axis, initial mass, and destruction velocity of chondrules used in the impact experiment.

\begin{tabular}{|c|c|c|c|}
\hline $\begin{array}{c}\text { chondrules } \\
\text { No. }\end{array}$ & $\begin{array}{c}\text { major } \\
\text { axis }(\mathrm{mm})\end{array}$ & mass $(\mathrm{g})$ & $V b(\mathrm{~m} / \mathrm{s})$ \\
\hline No. 1 & 1.86 & 0.0071 & $19 \pm 2$ \\
\hline No. 2 & 1.57 & 0.0044 & $76 \pm 15$ \\
\hline No. 3 & 1.52 & 0.0041 & $19 \pm 2$ \\
\hline No. 4 & 1.45 & 0.0036 & Lost \\
\hline No. 5 & 1.33 & 0.0022 & $28 \pm 2$ \\
\hline No. 6 & 1.13 & 0.0024 & $20 \pm 2$ \\
\hline No. 7 & 1.27 & 0.0024 & $45 \pm 15$ \\
\hline No. 8 & 1.13 & 0.0018 & $27 \pm 2$ \\
\hline No. 9 & 1.17 & 0.0018 & $54 \pm 15$ \\
\hline No. 10 & 1.07 & 0.0015 & $11 \pm 2$ \\
\hline No. 11 & 0.99 & 0.0014 & $54 \pm 15$ \\
\hline No. 12 & 0.87 & 0.0011 & $11 \pm 2$ \\
\hline No. 13 & 0.73 & 0.0007 & Lost \\
\hline No. 14 & 1.05 & 0.0009 & $17 \pm 2$ \\
\hline No. 15 & 0.90 & 0.001 & $49 \pm 15$ \\
\hline No. 16 & 1.00 & 0.0011 & $19 \pm 2$ \\
\hline No. 17 & 1.09 & 0.0009 & $67 \pm 15$ \\
\hline No. 18 & 0.88 & 0.0006 & $22 \pm 2$ \\
\hline No. 19 & 0.88 & 0.001 & Lost \\
\hline No. 20 & 0.86 & 0.001 & $72 \pm 15$ \\
\hline No. 21 & 0.86 & 0.0006 & $23 \pm 2$ \\
\hline No. 22 & 0.88 & 0.0008 & $28 \pm 2$ \\
\hline No. 23 & 0.92 & 0.0008 & $27 \pm 2$ \\
\hline No. 24 & 0.89 & 0.0008 & $20 \pm 2$ \\
\hline No. 25 & 0.81 & 0.0007 & $16 \pm 2$ \\
\hline No. 26 & 0.75 & 0.0005 & Lost \\
\hline No. 27 & 0.90 & 0.0007 & Lost \\
\hline No. 28 & 0.81 & 0.0006 & $28 \pm 2$ \\
\hline No. 29 & 0.86 & 0.0006 & Lost \\
\hline No. 30 & 0.82 & 0.0007 & $72 \pm 15$ \\
\hline No. 31 & 1.62 & 0.0061 & $20 \pm 2$ \\
\hline No. 32 & 2.55 & 0.0181 & $19 \pm 2$ \\
\hline No. 33 & 2.26 & 0.0094 & $22 \pm 2$ \\
\hline No. 34 & 2.85 & 0.0275 & $23 \pm 2$ \\
\hline No. 35 & 2.33 & 0.0057 & $19 \pm 2$ \\
\hline No. 36 & 2.19 & 0.0098 & $22 \pm 2$ \\
\hline No. 37 & 1.26 & 0.0015 & $20 \pm 2$ \\
\hline No. 38 & 2.12 & 0.0046 & $54 \pm 15$ \\
\hline No. 39 & 1.40 & 0.0027 & Lost \\
\hline No. 40 & 1.12 & 0.0017 & $17 \pm 2$ \\
\hline No. 41 & 1.12 & 0.0011 & $25 \pm 2$ \\
\hline No. 42 & 1.05 & 0.001 & $14 \pm 2$ \\
\hline No. 43 & 1.09 & 0.0011 & $49 \pm 15$ \\
\hline No. 44 & 0.81 & 0.0007 & $22 \pm 2$ \\
\hline No. 45 & 1.19 & 0.002 & $45 \pm 15$ \\
\hline No. 46 & 1.05 & 0.0009 & $19 \pm 2$ \\
\hline No. 47 & 1.31 & 0.0018 & $19 \pm 2$ \\
\hline No. 48 & 1.71 & 0.0051 & $20 \pm 2$ \\
\hline No. 49 & 1.17 & 0.0008 & Lost \\
\hline No. 50 & 2.12 & 0.0095 & $20 \pm 2$ \\
\hline No. 51 & 1.19 & 0.0017 & $72 \pm 15$ \\
\hline No. 52 & 1.45 & 0.0013 & $20 \pm 2$ \\
\hline No. 53 & 1.07 & 0.0015 & $23 \pm 2$ \\
\hline No. 54 & 1.17 & 0.0011 & $16 \pm 2$ \\
\hline No. 55 & 1.95 & 0.0056 & $22 \pm 2$ \\
\hline No. 56 & 1.21 & 0.0014 & $19 \pm 2$ \\
\hline No. 57 & 1.07 & 0.0015 & $22 \pm 2$ \\
\hline No. 58 & 1.50 & 0.0013 & $19 \pm 2$ \\
\hline No. 59 & 1.00 & 0.0005 & $28 \pm 2$ \\
\hline No. 60 & 1.24 & 0.0011 & $19 \pm 2$ \\
\hline No. 61 & 1.24 & 0.0011 & $17 \pm 2$ \\
\hline No. 62 & 0.93 & 0.0005 & $20 \pm 2$ \\
\hline No. 63 & 1.09 & 0.0006 & $49 \pm 15$ \\
\hline No. 64 & 0.88 & 0.0005 & $20 \pm 2$ \\
\hline No. 65 & 0.74 & 0.0004 & $49 \pm 15$ \\
\hline
\end{tabular}

\begin{tabular}{|c|c|c|c|}
\hline $\begin{array}{c}\text { chondrules } \\
\text { No. }\end{array}$ & $\begin{array}{c}\text { major } \\
\text { axis }(\mathrm{mm})\end{array}$ & mass $(\mathrm{g})$ & $V b(\mathrm{~m} / \mathrm{s})$ \\
\hline No. 66 & 0.71 & 0.0003 & $49 \pm 15$ \\
\hline No. 67 & 0.83 & 0.0004 & $27 \pm 2$ \\
\hline No. 68 & 1.09 & 0.0007 & $14 \pm 2$ \\
\hline No. 69 & 1.09 & 0.0006 & $25 \pm 2$ \\
\hline No. 70 & 0.86 & 0.0005 & Lost \\
\hline No. 71 & 0.69 & 0.0004 & $45 \pm 15$ \\
\hline No. 72 & 0.67 & 0.0004 & Lost \\
\hline No. 73 & 0.60 & 0.0003 & Lost \\
\hline No. 74 & 0.69 & 0.0003 & $22 \pm 2$ \\
\hline No. 75 & 0.74 & 0.0005 & Lost \\
\hline No. 76 & 1.12 & 0.0003 & $23 \pm 2$ \\
\hline No. 77 & 0.69 & 0.0004 & $25 \pm 2$ \\
\hline No. 78 & 0.81 & 0.0005 & Lost \\
\hline No. 79 & 0.67 & 0.0003 & $23 \pm 2$ \\
\hline No. 80 & 0.74 & 0.0002 & $27 \pm 2$ \\
\hline No. 81 & 0.69 & 0.0002 & $11 \pm 2$ \\
\hline No. 82 & 0.60 & 0.0003 & $27 \pm 2$ \\
\hline No. 83 & 0.52 & 0.0002 & $16 \pm 2$ \\
\hline No. 84 & 0.71 & 0.001 & $63 \pm 15$ \\
\hline No. 85 & 0.45 & 0.0001 & $22 \pm 2$ \\
\hline No. 86 & 1.81 & 0.0083 & $16 \pm 2$ \\
\hline No. 87 & 0.81 & 0.0005 & $14 \pm 2$ \\
\hline No. 88 & 1.12 & 0.0008 & $58 \pm 15$ \\
\hline No. 89 & 1.52 & 0.0032 & $17 \pm 2$ \\
\hline No. 90 & 0.88 & 0.0005 & $25 \pm 2$ \\
\hline No. 91 & 1.33 & 0.0016 & $23 \pm 2$ \\
\hline No. 92 & 1.17 & 0.0012 & $45 \pm 15$ \\
\hline No. 93 & 1.43 & 0.0023 & Lost \\
\hline No. 94 & 1.07 & 0.0009 & $23 \pm 2$ \\
\hline No. 95 & 1.12 & 0.002 & Lost \\
\hline No. 96 & 0.69 & 0.0003 & $45 \pm 15$ \\
\hline No. 97 & 1.36 & 0.0025 & $19 \pm 2$ \\
\hline No. 98 & 2.40 & 0.0127 & $27 \pm 2$ \\
\hline No. 99 & 1.26 & 0.0012 & $19 \pm 2$ \\
\hline No. 100 & 1.55 & 0.0036 & $20 \pm 2$ \\
\hline No. 101 & 1.26 & 0.0019 & $28 \pm 2$ \\
\hline No. 102 & 1.57 & 0.0019 & $20 \pm 2$ \\
\hline No. 103 & 0.62 & 0.0002 & $23 \pm 2$ \\
\hline No. 104 & 1.02 & 0.0009 & $20 \pm 2$ \\
\hline No. 105 & 1.40 & 0.0026 & $28 \pm 2$ \\
\hline No. 106 & 2.60 & 0.0178 & $20 \pm 2$ \\
\hline No. 107 & 2.09 & 0.0097 & $23 \pm 2$ \\
\hline No. 108 & 0.57 & 0.0002 & $45 \pm 15$ \\
\hline No. 109 & 1.79 & 0.0083 & $14 \pm 2$ \\
\hline No. 110 & 1.45 & 0.0024 & $22 \pm 2$ \\
\hline No. 111 & 1.38 & 0.0034 & $54 \pm 15$ \\
\hline No. 112 & 0.76 & 0.0005 & $16 \pm 2$ \\
\hline No. 113 & 0.86 & 0.0007 & $19 \pm 2$ \\
\hline No. 114 & 2.15 & 0.0137 & $19 \pm 2$ \\
\hline No. 115 & 0.76 & 0.0003 & $14 \pm 2$ \\
\hline No. 116 & 0.48 & 0.0002 & $54 \pm 15$ \\
\hline No. 117 & 0.95 & 0.0009 & $49 \pm 15$ \\
\hline No. 118 & 0.55 & 0.0002 & $11 \pm 2$ \\
\hline No. 119 & 1.43 & 0.0026 & $16 \pm 2$ \\
\hline No. 120 & 0.69 & 0.0004 & $28 \pm 2$ \\
\hline No. 121 & 1.12 & 0.0009 & Lost \\
\hline No. 122 & 0.88 & 0.0007 & $22 \pm 2$ \\
\hline No. 123 & 1.79 & 0.008 & $20 \pm 2$ \\
\hline No. 124 & 0.64 & 0.0001 & $20 \pm 2$ \\
\hline No. 125 & 1.21 & 0.0012 & $23 \pm 2$ \\
\hline No. 126 & 0.74 & 0.0003 & $45 \pm 15$ \\
\hline No. 127 & 1.07 & 0.0006 & $49 \pm 15$ \\
\hline No. 128 & 0.64 & 0.0005 & $49 \pm 15$ \\
\hline No. 129 & 0.67 & 0.0008 & $20 \pm 2$ \\
\hline No. 130 & 1.09 & 0.0011 & $28 \pm 2$ \\
\hline No. 131 & 1.71 & 0.0044 & $23 \pm 2$ \\
\hline
\end{tabular}




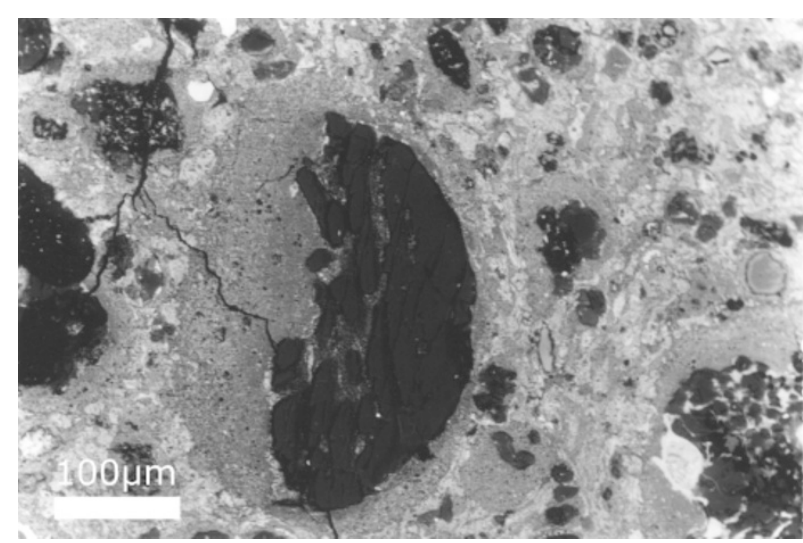

Fig. 1. A back-scattered electron (BSE) image of a broken chondrule with fine-grained rim in the CM chondrite Yamato-791198. The left side of the chondrule shows irregular morphology, while the remainder is smooth and rounded, suggesting that the left part of the chondrule was broken off after chondrule formation. The fine-grained rim enclosed the entire chondrule, suggesting that destruction took place prior to rim formation.

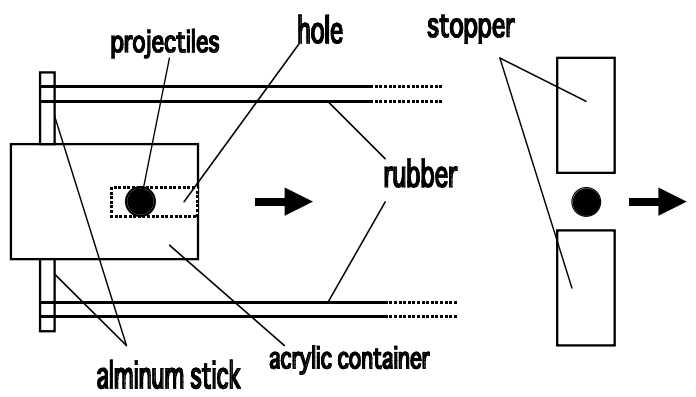

Fig. 2. The rubber gun launcher. The rubber gun is quite similar to that of a slingshot. The projectile is put into an acrylic container and then launched. The container impacts into a stopper on the way to the target and releases the projectile, which flies into a stainless steel target.

$2.6 \mathrm{~g} / \mathrm{cm}^{3}$ were launched using the rubber gun. Successive photographs of the flying glass spheres were taken using a high-speed strobe scope to determine spherule velocity. The relationship between the velocity of the sphere and the expansion of rubber is shown in Fig. 3. The uncertainty of the velocity measurements in the rubber gun experiments is $\pm 2 \mathrm{~m} / \mathrm{s}$ (Fig. 3). We then performed the experiments with the chondrules, but could not take photographs of the flying chondrules due to dark their surface color. Thus we assumed that the velocity of a chondrule is equal to that of the glass sphere with the same expansion of rubber. The validity of the assumption is explained as follows. The mass of an acrylic container, which moves with and accelerates a projectile (Fig. 2), is $18 \mathrm{~g}$, while the mass of the projectile such as the glass sphere $(0.17 \mathrm{~g})$ and a chondrule $(\sim 0.0024 \mathrm{~g})$ is very small relative to that of the container. Thus the velocity of the container is constant regardless of whether the projectile is a sphere or a chondrule. We assume that the difference in air friction between projectiles is negligible due to the short flying distance to the target. Consequently, the velocity of the glass sphere and that of the chondrule at the impact plane of the target is assumed to be the same as the launch velocity.

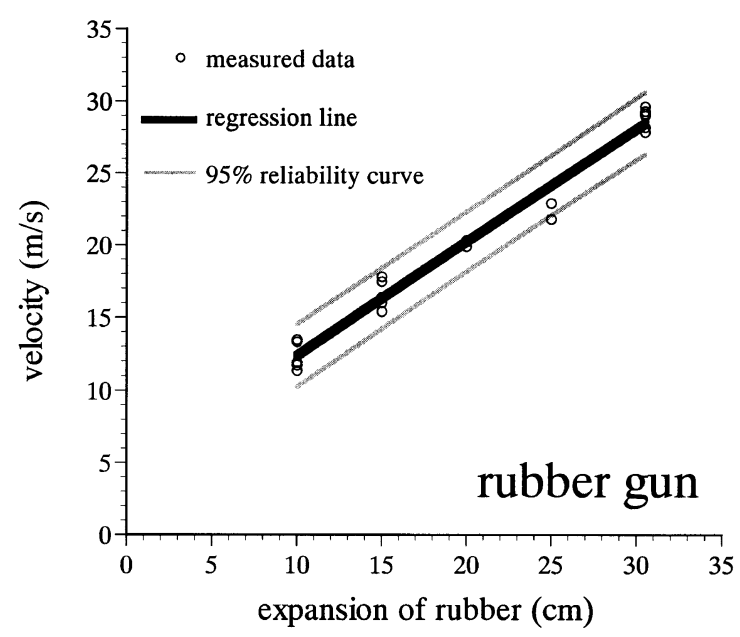

Fig. 3. The relationship between the rubber expansion and the glass sphere velocity. Velocity is proportional to the expansion of rubber from 10 to $30 \mathrm{~m} / \mathrm{s}$. The regression line shown is used for calibration of chondrule velocity and the uncertainty of the velocity measurement is approximately $\pm 2 \mathrm{~m} / \mathrm{s}$.

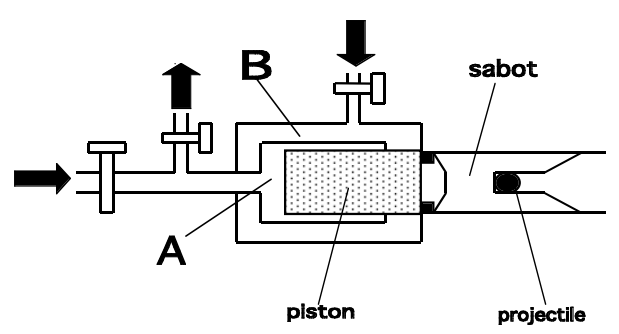

Fig. 4. The air gun launcher. $P_{\mathrm{A}}$ and $P_{\mathrm{B}}$ are the air pressures in sections $\mathrm{A}$ and $\mathrm{B}$, respectively. Initially, $P_{\mathrm{A}}$ is increased to a designated pressure to press the piston toward the sabot. Then, $P_{\mathrm{B}}$ is increased to a pressure not more than $P_{\mathrm{A}}$. To launch the sabot, $P_{\mathrm{A}}$ is released by opening a valve, which presses the piston backward, introduces high-pressure air to the back of the sabot, and accelerates it. The sabot impacts a stopper on the way to a stainless steel target and launches the projectile. The distance between the stopper and the target is $40 \mathrm{~cm}$.

The air gun consists of a launcher (Fig. 4), a recovery apparatus with a stainless steel target, and a laser for measuring the projectile velocity. Chondrules were difficult to detect with the laser because of their small size. As a result, the velocity of the chondrule was estimated from that of the glass spherule at the same external pressure. Again, this estimation is valid because the mass of the projectile, such as the glass spherule or the chondrule, is very small compared with that of a sabot (16 g) in the air gun (Fig. 4). We shot new glass spherule for velocity calibration every time we changed the external pressure $P_{\mathrm{B}}$ at the launcher part (Fig. 4) and the relationship between the velocity of a chondrule and the external pressure is shown in Fig. 5. The uncertainty in the velocity calibration of the air gun is \pm 15 $\mathrm{m} / \mathrm{s}$, which is relatively poor compared with that of the rubber gun (Fig. 5). The velocity decrease due to the air friction between the launcher and the target is calculated to be at most $20 \%$ for the smallest sample with $0.4 \mathrm{~mm}$ diameter.

2.2.2 Chondrule destruction experiment At first, all the Allende chondrule samples were shot using the rubber gun at the lowest velocity $(10 \pm 2 \mathrm{~m} / \mathrm{s})$. Then, the velocity 


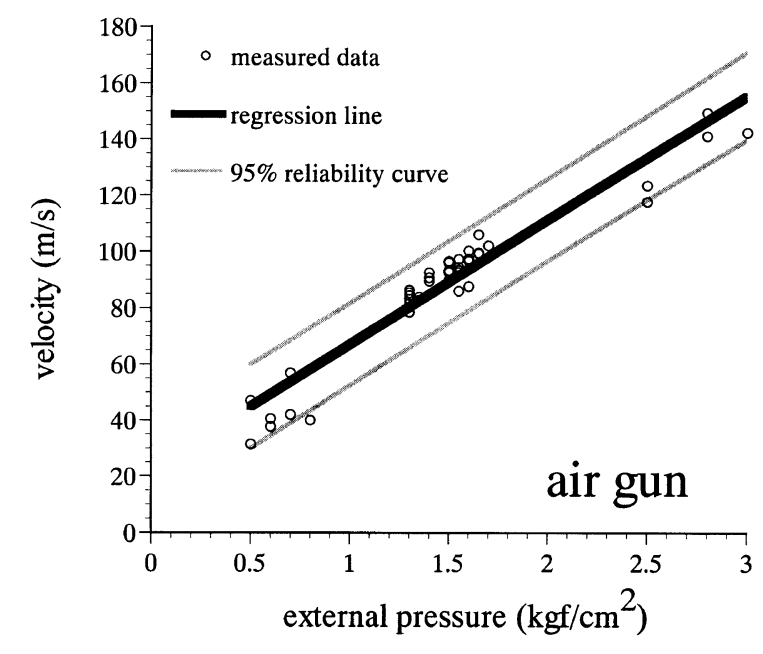

Fig. 5. The velocity of the glass sphere that increases in proportion to $P_{\mathrm{B}}$ in the air gun. The regression line shown is used for calibration of chondrule velocity and the uncertainty of the velocity measurement is approximately $\pm 15 \mathrm{~m} / \mathrm{s}$.

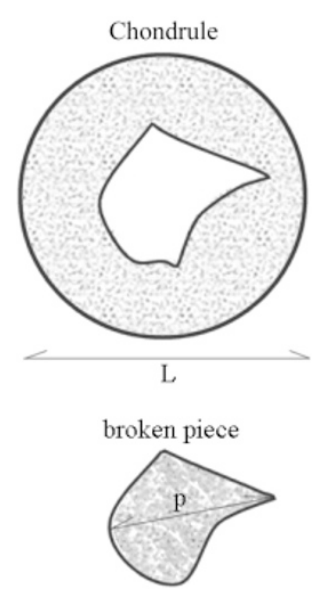

Fig. 6. A schematic drawing of the definition of destruction of a chondrule used in the impact experiment. A chondrule is defined as being broken when the longest dimension of the largest broken piece (p) exceeds $1 / 5$ of the initial diameter of the chondrule (L).

was increased step by step by extending the rubber in increments of $2 \mathrm{~m} / \mathrm{s}$ from 10 to $30 \mathrm{~m} / \mathrm{s}$, to the highest achievable velocity of the rubber gun. Chondrules were launched at increasing velocity until they were destroyed. We defined destroyed chondrules as those from which fragments with more than $1 / 5$ of the initial chondrule diameter were broken off (Fig. 6). The chondrules that were not destroyed by the rubber gun at the highest velocity $(30 \pm 2 \mathrm{~m} / \mathrm{s})$, were subsequently shot by the air gun. In the air gun experiment, the velocities were increased by raising the external pressure by $0.1 \mathrm{kgf} / \mathrm{cm}^{2}$ from $0.5(45 \pm 15 \mathrm{~m} / \mathrm{s})$ to $1.2 \mathrm{kgf} / \mathrm{cm}^{2}(76 \pm 15$ $\mathrm{m} / \mathrm{s}$ ), again the chondrules were launched until destruction.

2.2.3 Characterization of recovered chondrules Fragments from six chondrules, three of which were broken during low-velocity impact (No. 1, 109, and 110 in Table 1) and three of which were broken during high-velocity impact (No. 7, No. 45, and No. 111 in Table 1), were embedded in epoxy resin, polished using the micro-diamond paste.
They were analyzed with a scanning electron microscope (JEOL JSM-5800LV) and an electron probe microanalyzer (JEOL JXA-733 superprobe) equipped with a wave-lengthdispersive X-ray spectrometer (WDS), in order to investigate the textural differences between each chondrule and to observe cracked planes in chondrules.

\section{Result}

The results of the chondrule destruction experiments using the rubber and air guns are summarized in Fig. 7. The diagram shows that approximately $75 \%$ of the chondrules were destroyed by impacts with a velocity smaller than $30 \pm$ $2 \mathrm{~m} / \mathrm{s}$. Because no data was obtained between the highest velocity of the rubber gun $(30 \mathrm{~m} / \mathrm{s})$ and the lowest velocity of the air gun $(45 \mathrm{~m} / \mathrm{s})$, no increase was observed for this velocity range as seen in the cumulative curve in Fig. 7. From the lowest-velocity impact using the air gun, six of the chondrules that survived the impact with the highestvelocity of the rubber gun were broken. This suggests that the six chondrules may have been destroyed by an impact with velocity between 30 and $45 \mathrm{~m} / \mathrm{s}$. If so, the cumulative data curve in Fig. 7 would exhibit a smooth increase with no plateau. It is known that Allende meteorite contains various types of chondrules such as porphyritic and barred olivine types (McSween, 1977). Our results suggest that all types of chondrules are destroyed by impacts with the velocities of $76 \pm 15 \mathrm{~m} / \mathrm{s}$. The stainless steel target is used in our experiments, and the destructive velocity of the mutual collision of chondrules may be larger, which will be discussed in Subsection 4.2.

To investigate why some chondrules were broken by an impact at low-velocity, while others survived to a much higher velocity impact, internal textures of selected chondrules were documented by electron microscopy. Chon-

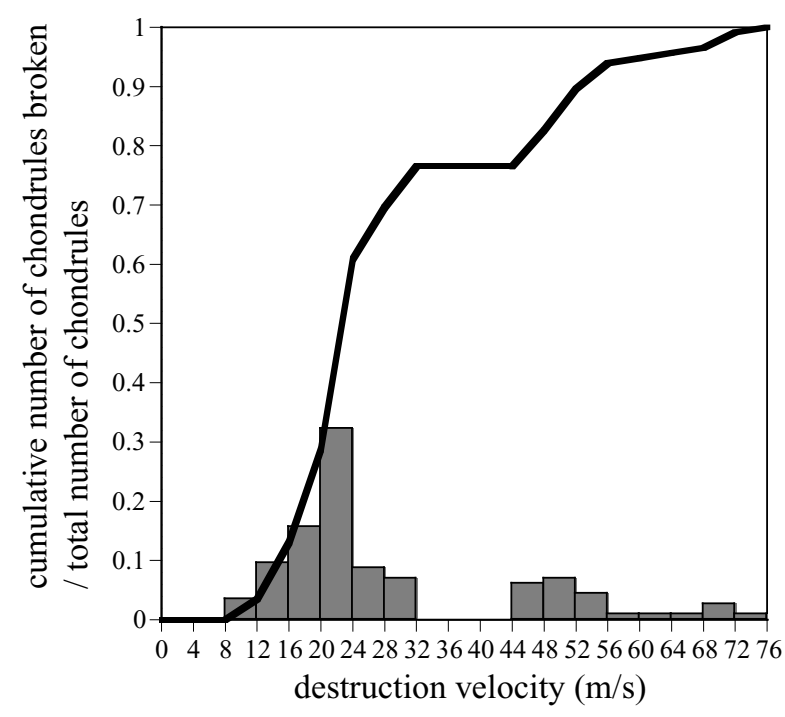

Fig. 7. Diagram showing the relationship between the destruction velocity and cumulative number of broken chondrules/total number of chondrules. The histogram indicates what fraction of chondrules were destroyed over the range of impact velocity. No data was obtained between the highest velocity of the rubber gun $(30 \mathrm{~m} / \mathrm{s})$ and the lowest velocity of the air gun $(45 \mathrm{~m} / \mathrm{s})$. 
drules those were broken by impacts at low velocities are classified into two types. The first type of chondrules (Figs. 8(a) and (b)) has pre-existing cracks filled with iron oxides such as magnetite, which are reopened again during the experimental impact. Chondrule No. 1 ( $V b=19 \pm$ $2 \mathrm{~m} / \mathrm{s}$, where $V b$ is the disruption velocity of the chondrule) is a porphyritic olivine chondrule with subhedral coarse olivine crystals embedded in mesostasis glass (Fig. 8(a)). In contrast, chondrule No. $109(V b=14 \pm 2 \mathrm{~m} / \mathrm{s})$ is a highly crystallized chondrule with a limited amount of glass (Fig. 8(b)). Both chondrules were broken at low impact velocity, indicating that chondrules containing cracks filled with iron oxide are not resistant to impact damage. The second type of chondrules is broken along the mesostasis glass. Chondrule No. $110(\mathrm{Vb}=22 \pm 2 \mathrm{~m} / \mathrm{s})$, a porphyritic olivine chondrule like chondrule No. 109, does not have iron oxide
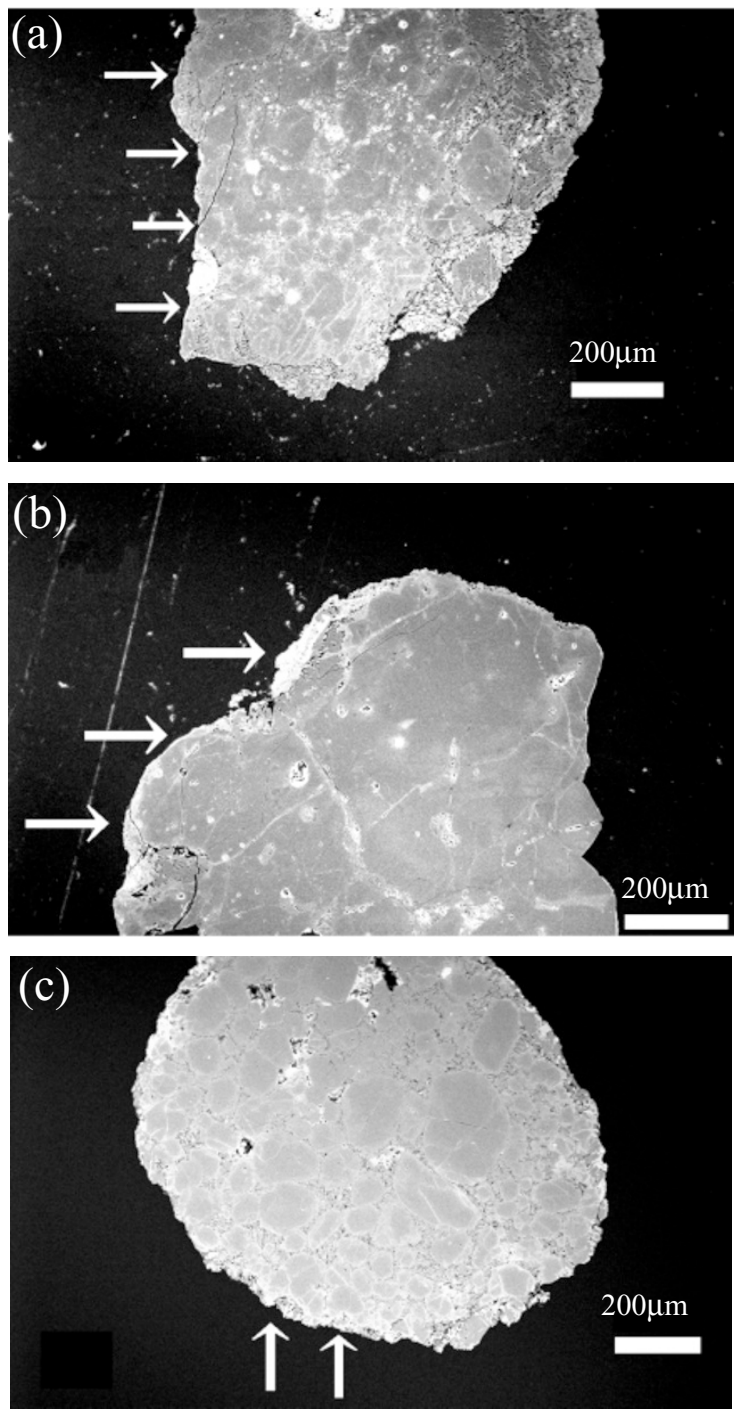

Fig. 8. BSE images of chondrules that were broken during the low velocity destruction experiment. (a) Chondrule No. 1 was broken along a crack filled with iron oxide (indicated by arrows). (b) Chondrule No. 109 consists exclusively of silicate crystals but was broken along a crack filled with iron oxide (indicated by arrows). (c) Chondrule No. 110 cracked in areas enriched in glass (indicated by arrows).



Fig. 9. A BSE image of a chondrule No. 45 destroyed by a high-velocity impact. The chondrule is composed almost exclusively of silicate minerals and has no pre-existing cracks filled with iron oxide.

filled cracks. This chondrule cracked along areas enriched in mesostasis glass (Fig. 8(c)).

Chondrules destroyed by a high-velocity impact commonly are well crystallized with very limited amounts of glass. Chondrule No. 45 ( $\mathrm{Vb}=45 \pm 15 \mathrm{~m} / \mathrm{s}$; Fig. 9) is a highly crystallized olivine and pyroxene chondrule. In this chondrule, both olivine and pyroxene were cracked during the experimental impact.

\section{Discussion}

\subsection{Secondary alteration effects on the destruction of chondrules}

Observations made by electron microscopes suggest that pre-existing iron oxide-filled cracks in chondrules significantly reduces their survivability during impacts. Well-crystallized chondrules are destroyed by a low-velocity impact if they have pre-existing cracks. Although Allende has experienced a limited degree of secondary processes in its meteorite parent body (e.g., Clayton and Mayeda, 1999; Scott et al., 1992), the iron oxide-filled cracks were produced by the combination of impact effects and aqueous alteration (Krot et al., 1998). This suggests that all of the chondrules used in the experiments were likely subject to secondary alteration that reduced the strength of these chondrules in experimental impacts. Thus if there are no pre-existing cracks in Allende chondrules, many chondrules might survive the experimental impacts with low velocities. However, we believe that the highest limit of the destruction velocity $76 \pm 15 \mathrm{~m} / \mathrm{s}$ does not change if there were no secondary alteration effects in Allende, because the chondrules broken during high-velocity impacts seem to be free of the alteration effects. Impact effects in Allende chondrules seem to be weak and variable between chondrules unlike other strongly shocked CV chondrules (Nakamura et al., 2000), because some chondrules in Allende show weak shock features, while others have no shock features (Scott et al., 1992). The chondrules with 
high-destruction velocity may have been those that were unaffected by shock on the meteorite parent body, because the chondrules with high-destruction velocity do not contain such iron oxide-filled cracks (Fig. 9). If there were preexisting shock features in chondrules, iron oxide has subsequently filled the shock-induced cracks as is observed in some chondrules (Figs. 8(a) and (b)). Therefore, we suggest that the chondrules with high-destruction velocity are free of pre-existing cracks and maintain survivability during impacts. On the basis of the interpretation stated above, we suggest that the upper limit of the velocity for chondrule destruction $76 \pm 15 \mathrm{~m} / \mathrm{s}$, if the target is as dense and rigid as the stainless steel used in our experiments (the destructive velocity of the mutual collision of chondrules may be larger, which will be discussed in the next subsection).

\subsection{Differences and similarities between experimental and natural impacts}

Chondrules consist mainly of silicates, which are brittle material and broken by the growth of cracks produced by an external force. Even by the weak force, this brittle material generates low density of lattice defects (dislocations), which weakens strength of chondrules. As the number of impact increases, dislocations and cracks may accumulate to a critical density enough for chondrule destruction. Thus, the destruction velocity of chondrules may differ between a single impact and multiple impacts.

It is not plausible that the impact velocity in the solar nebula increases step by step in an increment of $2 \mathrm{~m} / \mathrm{s}$ like in our experiment. This is a difference between experiment and nature, but there are similarities: (1) Chondrules might have experienced multiple impacts before the final destruction, and (2) the impact position on the surface of chondrules varies randomly between impacts. Chondrules commonly exhibit asymmetrical texture, and thus the minimum force to destroy chondrules must vary between the positions on chondrules. Thus, the impact position of chondrules is an important parameter for the destruction of chondrules. In our impact experiment we cannot control the impact position of chondrules and the impact positions must have varied between impacts, and the situation must be similar to the impact in the nebula.

We used the stainless steel target whose physical properties are different from those of chondrules. Thus, the stress exerted on chondrules in our experiment is different from that in the case of mutual collision of chondrules. Without a detailed knowledge of physical properties of chondrules, it is difficult to derive a precise relation of the critical destruction velocity of our experiment $V b$ and that of the mutual collision of chondrules $v_{b}$. In the limit of infinitly rigid and massive wall, the destruction velocity is considered to be half of that of the mutual collision of chondrules of an identical mass, since the wall is equivalent to the mirror symmetry plane of the mutual collision. Considering that the stainless steel target is fairly rigid and massive, we have $v_{b} \sim 2 \mathrm{Vb}$. On the other hand, a chondrule might collide with a massive and dense particle in the solar nebula. In this case, the destruction velocity would be nearly equal to that of our experimental value $V b$. Considering these uncertainties, the critical velocity of destruction of a mutual collision would be $76 \mathrm{~m} / \mathrm{s}$ to $152 \mathrm{~m} / \mathrm{s}$, where $76 \mathrm{~m} / \mathrm{s}$ is our experimental re- sult and $152 \mathrm{~m} / \mathrm{s}$ is the presumed critical value of mutual collision of chondrules with an identical mass.

\subsection{The origin of fragments of chondrules in unbrec- ciated chondrites}

We suggest four possible chondrule fragmentation processes in the solar nebula: 1) collisions between chondrules in the turbulent solar nebula, 2) collisions between chondrules in nebular shock waves, 3) collisions between chondrules in the bipolar flow, and 4) collisions of chondrules with larger bodies.

First, we investigate the possibility that fragments were produced by repeated collisions in the turbulent solar nebula. We calculate relations between the turbulent velocity and the relative velocity of chondrules in the nebula using equations derived by Völk et al. (1980). We here consider two different regions: (1) $2.4 \mathrm{AU}$ from the sun (the asteroid region) and (2) $0.065 \mathrm{AU}$ (the X-region). In the latter case, chondrules may have formed by activities (e.g., the magnetic reconnection) near the inner boundary of the nebula (Shu et al., 1996, 1997). We use two extreme nebular density models: (i) the Hayashi minimum density model (Hayashi, 1981; Hayashi et al., 1985), and (ii) the maximum density model, where the column density is given by the critical density of gravitational stability of the nebula; i.e. the Toomre parameter $Q \equiv c_{S} \Omega_{K} / \pi G \Sigma$ is equal to 1 (Toomre, 1964), where $\Sigma$ is the surface density, $c_{S}$ is the sound speed, $\Omega_{K}$ is the circular Keplerian angular velocity, and $G$ is the gravitational constant. Four cases are considered, (a) 2.4 AU in a Hayashi nebula (b) $2.4 \mathrm{AU}$ in a $Q=1$ nebula (c) 0.065 AU in a Hayashi nebula (d) $0.065 \mathrm{AU}$ in a $Q=1$ nebula. The parameters used in our calculations are the midplane temperature of the nebula $T_{c}$, the least wave number of eddy $k_{\min }$, and radii of the chondrules which collide each other: $r_{1}$ and $r_{2}$. In the case of $Q=1$ model, the midplane temperature is model dependent, but we here simply assume to be $T_{c}=1200 \mathrm{~K}$, which is the maximum temperature for chondrule survival, since the results do not depend on the temperature so sensitively. In the Hayashi model, the midplane temperature is given by

$$
T_{c}=280 \times(R / \mathrm{AU})^{-1 / 2} \mathrm{~K}
$$

(Hayashi, 1981; Hayashi et al., 1985), where $R$ is the distance from the sun. For the radii of two chondrules which collide each other, one is defined as $r_{1}=0.9 \mathrm{~mm}$ i.e. the mean radius of a chondrules in $\mathrm{CV}$ chondrites (Trude et al., 1978), and the other is treated as a parameter (we calculated for $r_{2}=1.0,3.0,5.0,7.0$ and $9.0 \mathrm{~mm}$ ). For these parameters, we calculated relationship between the root mean square turbulent velocity $\sqrt{\left\langle\delta v_{g}{ }^{2}\right\rangle}$ and the root mean square relative velocity of chondrules $\sqrt{\left\langle\Delta_{12} \delta v_{d}^{2}\right\rangle}$ from equations derived by Völk et al. (1980) with some revisions and assumptions. The details of the calculations are in the Appendix. The numerical results are shown in Fig. 10. Based on the model, the relative chondrule velocity increases as the radius $r_{2}$ increases, because a larger particle has a large relative velocity to the turbulent flow. In case (a), even if the relative velocities of chondrules with radii $r_{1}=0.9 \mathrm{~mm}$ (the mean radius of $\mathrm{CV}$ ) and $r_{2}=9.0 \mathrm{~mm}$ (one order of magnitude greater than $r_{1}$ ), the turbulent velocity $250 \mathrm{~m} / \mathrm{s}$ is required to reach the critical velocity of fragmentation, 76 

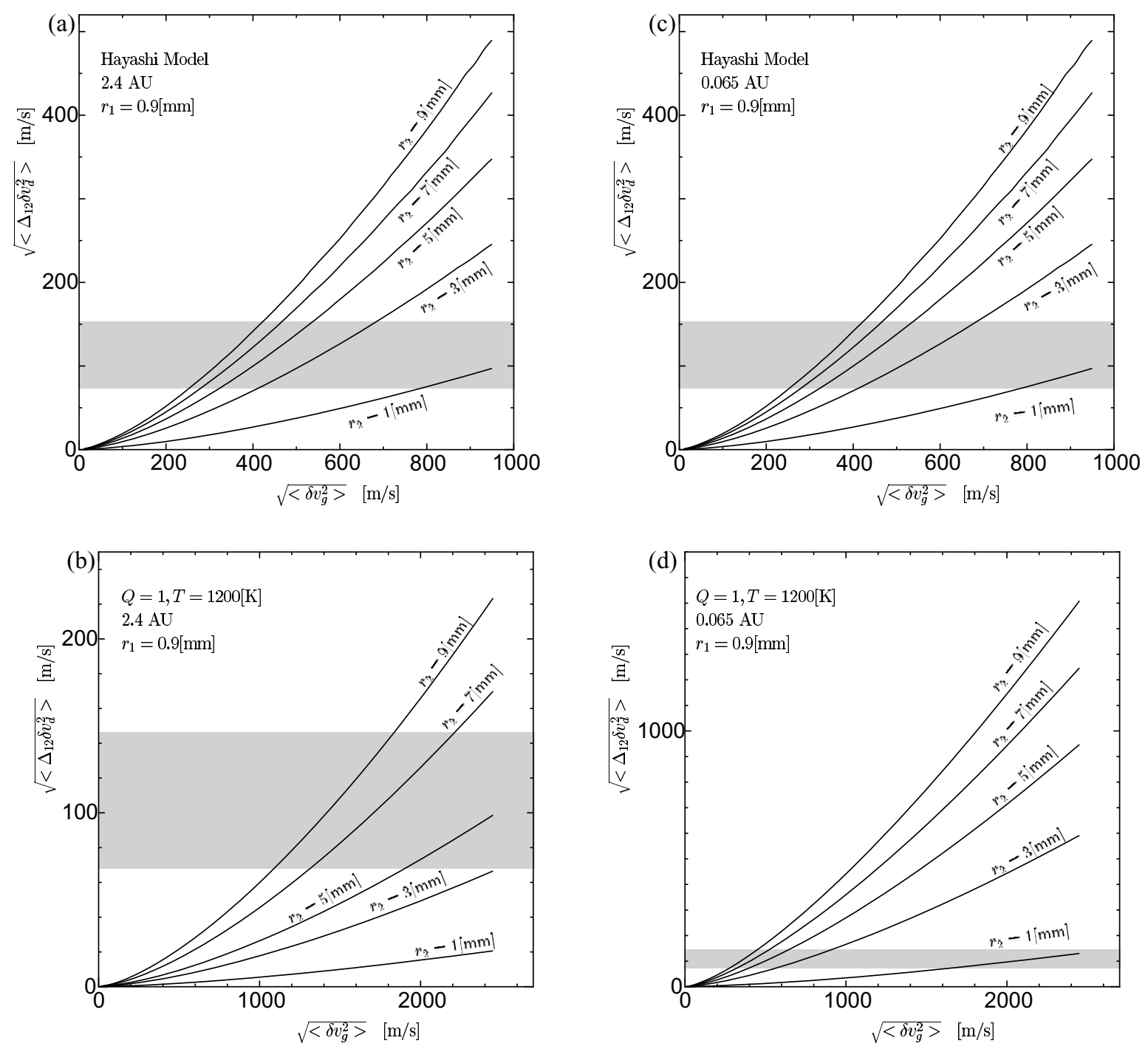

Fig. 10. The mean relative velocity $\sqrt{\left\langle\Delta_{12} \delta v_{d}{ }^{2}\right\rangle}$ of chondrules with radii $r_{1}=0.9 \mathrm{~mm}$ and $r_{2}=1.0,3.0,5.0,7.0,9.0 \mathrm{~mm}$ as a function of the mean turbulent velocity $\sqrt{\left\langle\delta v^{2}\right\rangle}$ for cases (a) $2.4 \mathrm{AU}-$ Hayashi model (b) $2.4 \mathrm{AU}-Q=1$ model (c) 0.065 AU—Hayashi model (d) 0.065 AU— $Q=1$ model. The maximum value of the horizontal axis (the mean turbulent velocity) is the sound velocity. Gray zones represent the critical velocity of fragmentation: 76 to $152 \mathrm{~m} / \mathrm{s}$.

$\mathrm{m} / \mathrm{s}$ as indicated by our experiments. The accretion velocity in the steady accretion disk is given by (Pringle, 1981)

$$
v_{R}=\frac{3 v_{t}}{2 R}
$$

where $v_{t}=\sqrt{\left\langle\delta v_{g}^{2}\right\rangle} H$ is the eddy viscosity coefficient, $H=\left(k T_{c} / m\right)^{1 / 2} \Omega_{K}^{-1}$ is the scale height of the solar nebula, $k$ is the Boltzmann constant, $m$ is the mean molecular mass of the nebular gas. The mass accretion rate is

$$
\dot{M}=2 \pi R \Sigma v_{R} .
$$

Thus, the root mean square turbulent velocity in the steady disk is expressed as

$$
\sqrt{\left\langle\delta v_{g}^{2}\right\rangle}=\frac{\dot{M} \Omega_{K}}{3 \pi \Sigma}\left(\frac{m}{k T_{c}}\right)^{1 / 2} .
$$

The turbulent velocities are several $\mathrm{cm} / \mathrm{s}$ for the $Q=1$ model and several $10 \mathrm{~cm} / \mathrm{s}$ for the Hayashi model, when the typical accretion rate $10^{-7} M_{\odot} \mathrm{yr}^{-1}$ for $T$ Tauri stars (Bertout et al., 1988). In case (b), the relative velocity is lower than for case (a), because the nebular density is higher so that the friction between chondrules and gas is stronger. Thus chondrule fragmentation in case (b) is less likely to occur. In the (c) and (d) X-region cases, it is also difficult to reach relative velocities exceeding the critical velocity as seen in Fig. 10. Since collisional velocities are distributed about the mean value, it is possible to have a collision with a velocity much higher than the mean value. However, the mean relative velocity of the chondrules is less than $1 \mathrm{~m} / \mathrm{s}$ for the all cases we calculated, so that they have an extremely low likelihood of reaching the impact destructive velocity $(76 \mathrm{~m} / \mathrm{s})$. For example, the probability is less than $10^{-2000}$ if the probability distribution is Gaussian. On the other hand, the mean collision time of chondrules is about 2.5 days in case (b) when collisional velocity is $152 \mathrm{~m} / \mathrm{s}$. If the duration of turbulence in the nebula was much greater, most of chondrules would have been destroyed. That is inconsistent with 
observations that there are lots of unbroken chondrules in chondrites. At least, it is not likely that the debris was produced in turbulence in the steadily accreting T Tauri nebula with $\dot{M} \sim 10^{-7} M_{\odot} \mathrm{yr}^{-1}$. Chondrules might be destructed in abrupt and/or localized strong turbulence.

Next, chondrules should have relative velocities, if they passed through a nebular shock wave (Hood and Horanyi, 1991, 1993). The relative velocities would have been very large $(\sim 1$ to $10 \mathrm{~km} / \mathrm{s})$. Chondrules should melt when the shock velocity and the optical depth were large (Hood and Horanyi, 1991, 1993). On the other hand, chondrules had merely be destroyed by mutual collisions, if the shock velocity was relatively small $(\lesssim 3 \mathrm{~km} / \mathrm{s})$. The mean collision time of chondrules is given by

$$
\tau_{\text {coll }}=\left[N \pi\left(r_{1}+r_{2}\right)^{2} \Delta_{12} v\right]^{-1},
$$

where $N$ is the chondrule number density, $\Delta_{12} v$ is the relative velocity of chondrules with radii $r_{1}$ and $r_{2}$. The stopping time of a chondrule due to gas friction is given by

$$
\tau_{f}=4 r \rho_{m} /\left(3 \rho_{g} v\right),
$$

where $r$ is the chondrule radius, $\rho_{g}$ is the gas density, $\rho_{m}$ is the material density of a chondrule, and $v$ is the chondrule velocity relative to the gas if the velocity $v$ is supersonic ( $v$ is replaced by $4 v_{\text {th }} / 3$ if the velocity is subsonic and the mean free path of the gas molecules $l_{g}$ is larger than $r$, where $v_{\text {th }}$ is the mean molecular velocity of the gas). The ratio of the collision time $\tau_{\text {coll }}$ to the stopping time $\tau_{f}$ is written

$$
\tau_{\text {coll }} / \tau_{f}=f^{-1}\left(\frac{r}{r_{1}+r_{2}}\right)^{2}\left(\frac{\Delta_{12} v}{v}\right)^{-1}
$$

where $f$ is the dust to gas mass ratio:

$$
f=\frac{N(4 \pi / 3) r^{3} \rho_{m}}{\rho_{g}} .
$$

For equilibrium between the dust settling and the turbulent mixing, $f \sim 1$ (Sekiya, 1998). Thus, the order of magnitude calculation with $r \sim r_{1}+r_{2}$, and $v \sim \Delta_{12} v$ gives $\tau_{\text {coll }} / \tau_{f} \sim 1$; that is, a considerable part of chondrules would be destroyed if a shock wave passed through the midplane of the solar nebula.

Furthermore, there is a possibility of fragmentation by mutual collision of chondrules, which were lifted by the bipolar flow (Liffman and Brown, 1996). However, it is difficult to estimate the relative velocity and the collision frequency of chondrules, since the velocity field of bipolar wind near the disk surface is not known in detail at present. This problem is left for future investigations.

Lastly, we examine collisions of chondrules with objects on the order of a meter in size or larger (Weidenschilling, private communication). When the friction time of the object is almost equal to the Kepler angular frequency (e.g., the diameter of the object is $1 \mathrm{~m}$ at $2.4 \mathrm{AU}$ in the minimum mass solar nebula), the object drifts radially at the highest velocity of $v_{K} \eta=54 \mathrm{~m} / \mathrm{s}$, where $v_{K}$ is the circular Kepler velocity, and

$$
\eta=-\frac{\partial P}{\partial \ln R} /\left(2 v_{K} \rho_{g}\right)
$$

where $P$ is the gas pressure (Adachi et al., 1976; Weidenschilling, 1977). Further, the relative transverse velocity between mm-sized chondrule and a body larger than $1 \mathrm{~m}$ is also equal to $v_{K} \eta$ (Adachi et al., 1976; Weidenschilling, 1977). Thus, if a chondrule collides with an object larger than $1 \mathrm{~m}$, the chondrule might be destroyed, if the object is as hard as the steel target of our experiment. On the other hand, chondrules may not be destroyed if the body were conglomerate of dust and chondrules and soft enough.

\section{Conclusion}

We have carried out collisional destruction experiment of chondrules taken from the Allende CV3 chondrite. A stainless steel target was used. The velocity of chondrule destruction distributes from a very small velocity $(\lesssim 10 \mathrm{~m} / \mathrm{s})$ to $76 \mathrm{~m} / \mathrm{s}$. Observation by electron microscopy showed that two types of chondrules were destroyed by low-velocity impacts: the first type has pre-existing cracks filled with ironoxides along which it was cracked again, and the second one is poorly crystallized with fine-grained silicates set in glass. On the other hand, relatively well-crystallized chondrules were destroyed by impacts at high velocity. If there were no pre-existing cracks which were presumed to have been made in secondary alteration, many chondrules would survive the experimental impact at low velocities. Considering the difference of our experiment with a stainless steel target from the mutual collision of chondrules, the upper limit of the destruction velocity is estimated to be $152 \mathrm{~m} / \mathrm{s}$. Using the chondrule destruction velocity, we theoretically examined the condition of the solar nebula in the periods when chondrules were destroyed. The results suggest that destruction of chondrules might have taken place by collisions between chondrules in abrupt and/or localized strong turbulence, or by the passage of the shock wave through the nebular midplane, or by collisions between chondrules and objects larger than $1 \mathrm{~m}$ in the laminar solar nebula.

Acknowledgments. We would like to thank Dr. Fujiwara of the Institute of Space and Astronautical Science for providing the strobe scope, Dr. Hanada of Kyushu University for giving us access to the air gun, Dr. Weidenschilling of Planetary Science Institute for his valuable comments, and Dr. Blum at Friedrich-SchillerUniversität Jena, and an anonymous reviewer for their critical review of the manuscript.

\section{Appendix A. Collisional Velocity of Chondrules in a Turbulent Nebula}

We assume that the energy spectrum of the turbulence is given by the Kolmogorov spectrum:

$$
P(k)=\frac{2\left\langle\left(\delta v_{g}\right)^{2}\right\rangle}{3 k_{0}}\left(\frac{k}{k_{0}}\right)^{-5 / 3},
$$

where $k$ is the wave number of a turbulent eddy, $k_{0}$ is the wave number of the largest eddy. We assume $k_{0}=2 \pi / H$, where $H$ is the scale height of the solar nebula. The lifetime of the eddy with wave number $k$ is given by equation (17) of Völk et al. (1980):

$$
\tau_{k}=\frac{1}{k(k P(k))^{1 / 2}},
$$


The response of a chondrule to the gas motion is characterized by the friction time:

$$
\tau_{f}= \begin{cases}r \rho_{m} /\left(v_{\mathrm{th}} \rho_{g}\right) & \text { for } r<l_{g} \\ 2 r^{2} \rho_{m} /\left(9 \rho_{g} \nu\right) & \text { for } r>l_{g},\end{cases}
$$

where $r$ and $\rho_{m}$ are the radius and material density of the chondrule, $v_{\text {th }}$ and $l_{g}$ are the mean thermal velocity and mean free path of the gas molecules, and $\rho_{g}$ is the gas density, and $v=v_{\text {th }} l_{g} / 2$ is the kinematic viscosity of the gas.

The wave number $k^{*}$ of the eddy for which a chondrule responds most well is given by equation (9) of Völk et al. (1980):

$$
\frac{1}{\tau_{f}}=\frac{1}{\tau_{k^{*}}}+k^{*} V_{\mathrm{rel}}\left(k^{*}\right),
$$

where the relative velocity of the chondrule and the eddy with wave number $k$ is given by equation (15) of Völk et al. (1980):

$$
\left[V_{\text {rel }}(k)\right]^{2}=\left\{\begin{array}{c}
\left(V_{L}\right)^{2}+\int_{k_{0}}^{\operatorname{Min}\left\{k^{*}, k\right\}} d k^{\prime} P\left(k^{\prime}\right) \frac{\tau_{f}}{\tau_{k^{\prime}}+\tau_{f}} \\
\quad+\int_{k^{*}}^{\operatorname{Max}\left\{k^{*}, k\right\}} d k^{\prime} P\left(k^{\prime}\right) ; k^{*}>k_{0} \\
\left(V_{L}\right)^{2}+\int_{k_{0}}^{k^{*}} d k^{\prime} P\left(k^{\prime}\right) ; k^{*}<k_{0} .
\end{array}\right.
$$

We assume that the constant part of the relative velocity $V_{L}$ (i.e., the mean relative velocity of chondrules to gas) is equal to zero.

The mutual velocity of chondrules, whose response times are $\tau_{f}(1)$ and $\tau_{f}(2)$ [with corresponding values of $k^{*}$ are denoted by $k^{*}(1)$ and $\left.k^{*}(2)\right]$ in turbulent nebula is calculated from equation (19) of Völk et al. (1980):

$$
\begin{aligned}
\left\langle\left(\Delta_{12} \delta v a\right)^{2}\right\rangle= & \int_{k_{0}}^{\operatorname{Min}\left\{k^{*}(1), k^{*}(2)\right\}} d k P(k) \\
& \cdot \frac{\tau_{k}\left(\tau_{f}(1)-\tau(2)\right)^{2}}{\left[\tau_{k}+\tau_{f}(1)\right]\left[\tau_{k}+\tau_{f}(2)\right]\left[\tau_{f}(1)+\tau_{f}(2)\right]} \\
& +\int_{\operatorname{Min}\left\{k^{*}(1), k^{*}(2)\right\}}^{\operatorname{Max}\left\{k^{*}(1), k^{*}(2)\right\}} d k P(k) \\
& \cdot \frac{\tau_{k}}{\left.\tau_{k}+\operatorname{Min}\left\{\tau_{f}(1), \tau_{f}(2)\right\}\right]} \\
& +\int_{k^{*}(1)}^{k_{\lambda}} d k P(k) \frac{\tau_{k}}{\tau_{k}+\tau_{f}(1)} \\
& \cdot \frac{\operatorname{arctg}\left\{\frac{k V_{\mathrm{rel}}(k) \tau_{k} \tau_{f}(1)}{\tau_{k}+\tau_{f}(1)}\right\}}{\left\{\frac{k V_{\mathrm{rel}}(k) \tau_{k} \tau_{f}(1)}{\tau_{k}+\tau_{f}(1)}\right\}} \\
& +\int_{k^{*}(2)}^{k_{\lambda}} d k P(k) \frac{\tau_{k}}{\tau_{k}+\tau_{f}(2)} \\
& \cdot \frac{\operatorname{arctg}\left\{\frac{k V_{\mathrm{rel}}(k) \tau_{k} \tau_{f}(2)}{\tau_{k}+\tau_{f}(2)}\right\}}{\left\{\frac{k V_{\mathrm{rel}}(k) \tau_{k} \tau_{f}(2)}{\tau_{k}+\tau_{f}(2)}\right\}} .
\end{aligned}
$$

Here we have changed the upper limit of integral of third and fourth terms from $\infty$ in the original paper to $k_{\lambda}=2 \pi / \lambda$, where $\lambda=\left(v^{3} / \epsilon\right)^{1 / 4}$ is the Kolmogorov length scale, and $\epsilon$ is the energy dissipation rate per unit mass of the turbulent gas, which is given by $\epsilon=V^{3} / L$. $V$ and $L$ are the velocity and length scale of the largest eddy, which are assumed to be equal to $\sqrt{\left\langle\left(\delta v_{g}\right)^{2}\right\rangle}$ and $H$, respectively.

\section{References}

Adachi, I., C. Hayashi, and K. Nakazawa, The gas drag effect on the elliptic motion of a solid body in the primordial solar nebula, Progr. Theor. Phys., 56, 1756-1771, 1976.

Bertout, C., G. Basri, and J. Bouvier, Accretion disks around T Tauri stars, Astrophys. J., 330, 350-373, 1988.

Bunch, T. E. and R. S. Rajan, Meteorite regolithic breccias, in Meteorites and the Early Solar System, edited by J. F. Kerridge and M. S. Matthews, pp. 144-164, Univ. Arizona Press, Tucson, 1988.

Clayton, R. N. and T. K. Mayeda, Oxygen isotope studies of carbonaceous chondrites, Geochim. Cosmochim. Acta, 63, 2089-2104, 1999.

Gooding, J. L. and K. Keil, Relative abundances of chondrule primary textural types in ordinary chondrites and their bearing on conditions of chondrule formation, Meteoritics, 16, 17-43, 1981.

Hayashi, C., Structure of the solar nebula, growth and decay of magnetic fields and effects of magnetic and turbulent viscosities on the nebula, Progr. Theor. Phys. Suppl., 70, 35-53, 1981.

Hayashi, C., K. Nakazawa, and Y. Nakagawa, Formation of the Solar System, in Protostars and Planets II, edited by D. C. Black and M. S. Matthews, pp. 1100-1153, Univ. Arizona Press, Tucson, 1985.

Hewins, R. H., Retention of sodium during chondrule melting, Geochim. Cosmochim. Acta, 55, 935-942, 1991.

Hood, L. L. and M. Horanyi, Gas dynamic heating of chondrule precursor grains in the solar nebula, Icarus, 93, 259-269, 1991.

Hood, L. L. and M. Horanyi, The nebular shock wave model for chondrule formation: One-dimensional calculations, Icarus, 106, 179-189, 1993.

King, T. V. V. and E. A. Elbert, Grain size and petrography of C2 and C3 carbonaceous chondrites, Meteoritics, 13, 47-72, 1978

Krot, A. N., M. I. Petaev, E. R. D. Scott, B. G. Choi, M. E. Zolensky, and K. Keil, Progressive alteration in CV3 chondrites: More evidence for asteroidal alteration, Meteoritics and Planet. Sci., 33, 1065-1085, 1998.

Liffman, K. and M. J. I. Brown, The Protostellar jet model of chondrule formation, in Chondrules and the Protoplanetary Disk, edited by R. H. Hewins, R. H. Jones, and E. R. D. Scott, pp. 285-302, Cambridge Univ. Press, Cambridge, 1996.

McSween, H. Y., Petrographic variations among carbonaceous chondrites of the Vigarano type, Geochim. Cosmochim. Acta, 41, 1777-1790, 1977.

Metzler, K., A. Bischoff, and D. Stöffler, Accretionary dust mantles in CM chondrite: Evidence for solar nebula process, Geochim. Cosmochim. Acta, 56, 2873-2897, 1992.

Nakamura, T., K. Tomeoka, N. Takaoka, T. Sekine, and H. Takeda, Impactinduced textural changes of CV carbonaceous chondrites: Experimental reproduction, Icarus, 146, 289-300, 2000.

Pringle, J. E., Accretion discs in astrophysics, Ann. Rev. Astron. Astrophys., 19, 137-162, 1981.

Scott, E. R. D., K. Keil, and D. Stöffler, Shock metamorphism of carbonaceous chondrites, Geochim. Cosmochim. Acta, 56, 4281-4293, 1992.

Sekiya, M., Quasi-equilibrium density distributions of small dust aggregations in the solar nebula, Icarus, 133, 298-309, 1998.

Sekiya, M. and T. Nakamura, Condition for the formation of the compound chondrules in the solar nebula, Proc. NIPR Symp. on Antarctic Meteorites, 9, 208-217, 1996.

Shu, F. H., H. Shang, and T. Lee, Toward an astrophysical theory of chondrites, Science, 271, 1545-1552, 1996.

Shu, F. H., H. Shang, A. E. Glassgold, and T. Lee, X-rays and fluctuating X-winds from protostars, Science, 277, 1475-1479, 1997.

Steele, I. M., Primitive material surviving in chondrites: mineral grains, in Meteorites and the Early Solar System, edited by J. F. Kerridge and M. S. Matthews, pp. 808-818, Univ. Arizona Press, Tucson, 1988.

Toomre, A., On the gravitational stability of a disk of stars, Astrophys. J., 139, 1217-1238, 1964.

Völk, H. J., F. C. Jones, G. E. Morfill, and S. Röser, Collisions between grains in a turbulent gas, Astron. Astrophys., 85, 316-325, 1980.

Wasson, J. T., Chondritic meteorites as products of the solar nebula, in Meteorites, pp. 136-154, Springer-Verlag, New York, 1974.

Weidenschilling, S. J., Aerodynamics of solid bodies in the solar nebula, Mon. Not. R. Astron. Soc., 180, 57-70, 1977.

Weisberg, M. K. and M. Prinz, Fayalitic olivine in CV3 chondrite matrix and dark inclusions: A nebula origin, Meteoritics and Planet. Sci., 33, 1087-1099, 1998.

T. Ueda, Y. Murakami, N. Ishitsu, H. Kawabe, R. Inoue, T. Nakamura, M. Sekiya (e-mail: sekiya@geo.kyushu-u.ac.jp), and N. Takaoka 